\title{
IL-17A/IL-17F Double KO Mice Are Resistant to Lipopolysaccharide Induced Endotoxic Shock
}

\author{
Anwarul Haque ${ }^{1,2}$, Chiaki Kajiwara², Tetsuya Matsumoto ${ }^{1}$, Yoshikazu Ishii ${ }^{2}$ and Kazuhiro Tateda ${ }^{2}$ \\ ${ }^{1}$ Department of Microbiology, Tokyo Medical University, Tokyo, Japan \\ ${ }^{2}$ Department of Microbiology and Infectious Diseases, Toho University School of Medicine, Tokyo, Japan
}

Corresponding author: Anwarul Haque, Department of Microbiology, Tokyo Medical University, 6-1-1 Shinjuku, Shinjuku-ku, Tokyo 160-8402, Japan, Tel: +81-3-3351-6141(Ext. 241); Fax: +81-3-3351-6160; E-mail: haqamk@gmail.com

Received Date: April 26, 2017; Accepted Date: May 03, 2017; Published Date: May 15, 2017

Copyright: (c) 2017 Haque A, et al. This is an open-access article distributed under the terms of the Creative Commons Attribution License, which permits unrestricted use, distribution, and reproduction in any medium, provided the original author and source are credited.

Citation: Haque A, Kajiwara C, Matsumoto T, et al. IL-17A/IL-17F Double KO Mice Are Resistant to Lipopolysaccharide Induced Endotoxic Shock. Eur Exp Biol 2017, 7:14.

\section{Abstract}

Aim: IL-17 family members, IL-17A and -17F are proinflammatory cytokines important for host immune modulation in infection and inflammatory diseases conditions. IL-17A has been shown playing a critical role in defense against bacterial infections and IL-17AF deficient mice (DKO) reported less protective. However, the role of IL-17 in endotoxic shock is largely undefined.

Materials and Methods: Wild and DKO mice were intraperitoneally lipopolysaccharide (LPS) administered and their survival status was recorded. Neutrophil, -T cells in peritoneal fluids and pro- and anti-inflammatory cytokines, chemokines in serum in endotoxic wild and DKO mice were evaluated.

Results: In this study, we observed a higher mortality rate in wild than DKO, in intraperitoneal LPS induced shock. Mortality was observed in correlation with increased proinflammatory cytokines and chemokines. We also observed a significant rise of -T cells in peritoneal cavity by LPS in wild, which is known to be a most potent source for IL-17 release and neutrophil recruitment at the site of infection. Neutrophil recruitment was shown as a protective phenomenon in murine in bacterial infection, but the same phenomenon was not observed in LPS induced sepsis.

Conclusions: These findings suggest that neutrophil recruitment at infection site may be beneficial in case of direct bacterial exposure, but not in endotoxin exposure to host tissue. This study shades a comprehensive understanding of IL-17A/F functions in acute peritonitis followed by endotoxic shock that could be beneficial for selection of infection for IL-17 directed therapy.

Keywords: IL-17A; IL-17F; Endotoxic shock; Neutrophils; T cells; Cytokines

\section{Introduction}

In recent years, IL-17 probably the most discussed cytokine in infectious disease field. This pro-inflammatory cytokine is regulated by the differentiation of naive T cells to Th17 cells implied by receiving signals from a wide variety of inflammatory conditions like autoimmune diseases, chronic inflammatory diseases and pathogenic infections [1]. IL-17 cytokine family comprises of six members including signatory IL-17A. And IL-17F is the most closely homologue with IL-17A by structure, source and functions. These two cytokines share same receptors and are usually discussed together in disease conditions. Studies revealed that upon release of cytokine IL-17A, it individually or in conjunction with IL-17F exerts protective functions for host against certain pathogens at epithelial and mucosal barriers [2-4]. They are important for the recruitment of neutrophils at the site of infection that ultimately limits spreading of bacteria and clear it off from the site of infection. Neutrophil dependent immune modulating properties of IL-17A and IL-17F are evident in both extracellular and intracellular bacterial infections [5-10]. On the contrary, a defect in IL-17A or in IL-17A/F induction in response to infection results increased bacterial dissemination, correlating with reduced inflammatory mediators and neutrophil recruitment in hosts [11]. Same study also focused on existence of functionally diverse pathogenic and non-pathogenic subpopulations of Th17 cells under specific clinical conditions. IL-17 functions widely investigated in lung infections, but not in peritoneal infection, particularly in acute endotoxic peritonitis.

Bacterial peritonitis remains a serious complication, especially after abdominal surgery and continuous peritoneal dialysis, influencing patient's expanses and mortality [12]. Peritoneum is the largest avascular organ in human body implicated for easy dissemination of peritoneal infection to general septicemia. However, published results about role of IL-17A/F in peritonitis are not constant. In cecum ligation and puncture (CLP) induced peritonitis, when a group showed IL-17 function is beneficial for survival [13], then other group showed that neutralization of peritoneal IL-17A bought successes to down mortality rate in their experimental mice [14]. Discrepancy in results in these studies may be related to the different level of inflammatory 
status in experimental models, since severity of sepsis in CLP models are not constant. On the contrary, use of a single dose of endotoxin to induce peritonitis in murine models could be a better option to compromise the discrepancy about severity of inflammatory reactions in experimental models. Furthermore, since bacterium itself is not harmful, but its endotoxin, so the host immune mechanism mediated by bacterial toxin could be different than that of immunity induced by direct bacterial exposure in host. This understanding may provide new window for immune modulation and infection treatment. Here we created LPS induced peritonitis in wild and in IL-17AF KO (double $\mathrm{KO}, \mathrm{DKO}$ ) mice to understand the function of IL-17AF in murine endotoxic shock.

\section{Materials and Methods}

All the experiments were conducted according to Toho University School of Medicine ethical guidelines for animal experiments after an approval of protocol by the institutional animal care and use committee (approval number 14-52-220).

\section{Animals and their survivals}

Specific-pathogen-free 7- to 8-week-old female BALB/c mice (wild) were purchased from Charles River Laboratories (Kanagawa, Japan) and were housed with standard laboratory food, water ad libitum, under specific-pathogen-free conditions within the animal care facility in Toho University School of Medicine, Tokyo, until the end of experiments. A constant temperature $\left(27^{\circ} \mathrm{C}\right)$, humidity $(65 \%)$ and $12 \mathrm{hrs} \mathrm{light/dark} \mathrm{cycle}$ was maintained in the facility. IL-17A/IL-17F double knockout (DKO) female mice (II17a-/-\|17f-/-) on BALB/c genetic background were previously established at the Institute of Medical Science, University of Tokyo $[15,16]$, were also being hosed in our institute under same conditions. First, LD50 dose of lipopolysaccharide (LPS) was determined for experimental models. LPS that purified from Escherichia coli 055:B5 was purchased from Sigma-Aldrich Japan and was dissolved in sterile $0.9 \% \mathrm{NaCl}$ solution (normal saline) at a required concentration. Wild mice were randomly divided in 5 groups where each group contained 8-10 mice. All mice were anaesthetized by exposing to isoflurane (1\%) for short duration, weighted and each group intraperitoneally LPS administered at either dose of 4, 8, 10, 12 or $15 \mathrm{mg} / \mathrm{kg}$ bd. wt. Mice were monitored for signs of illness and/or unusual behavior every 12 hourly for 120 hrs. Endpoints of survival inspection were determined by existence of classical signs of distress such as anorexia, hunching, prostration, impaired motility, labored breathing and ruffled hair-coat and at endpoints, mice were humanely euthanized using isoflurane (5\%) anesthesia followed by cervical dislocation. Endpoints were considered as 'non-survivor' and thus survival proportions in different wild groups were statistically calculated to determine their LD50 dose of LPS. Same endpoint criteria and animal sacrifice protocol were followed in all survival experiments in this study. Next, age matched wild and DKO mice were intraperitoneally LPS administered at a determined LD50 dose. Outcome of induced shock in models were observed and recorded 12 hourly for $120 \mathrm{hrs}$. Survival proportion in endotoxic shock models, following acute peritonitis, were examined by minimum three sets of individual experiments.

\section{Biological samples from endotoxic shock models}

A total of 5 mice from each wild and DKO mice were intraperitoneally $5 \mathrm{ml}$ PBS administered and their peritoneal lavage were collected, as a control samples. Other groups of mice containing 5 wild and 5 DKO were induced peritonitis by intraperitoneally LPS administration at a dose of LD50 and peritoneal lavages were collected after $12 \mathrm{hrs}$ of LPS administration following the same way. Immediately after obtaining the peritoneal fluid, samples were processed for flowcytometric analysis to count the number of neutrophils and T-cells in fluids.

As a separate set of experiments, wild and DKO mice were randomly selected and each group further divided into 6 subgroups, where each sub-group included 7 to 10 mice. These mice were challenged with LPS following same method, that of challenged for survival experiments. Mice were anesthetized by subcutaneous ketamine/xylazine (100: $10 \mathrm{mg} \mathrm{kg}^{-1}$ body weight) administration and blood samples were collected from these groups of mice by inferior-venaceva (IV) puncture at $0,3,6,12$, 18 and $24 \mathrm{hrs}$ of LPS administration. After collection, blood were allowed to clot and at $4^{\circ} \mathrm{C}$ centrifuged with $2400 \mathrm{Xg}$ for $15 \mathrm{~min}$ to obtain serum from blood samples, which were stored in aliquots at $-80^{\circ} \mathrm{C}$ for later assays. All anesthetized mice were sacrificed by cervical dislocation.

\section{Assessment by flowcytometry and ELISA}

The antibodies for detection of neutrophils and -T cells were purchased from Biolegand Inc., Japan. Neutrophils and T-cells in peritoneal lavage were counted after erythrocyte lysis and were stained with PerCP/Cy5.5-conjugated anti-mouse Ly6G (clone: 1A8) and FITC-conjugated anti-mouse TCR (clone:GL3), respectively. Cell numbers were calculated in percentage by $B D$ FACS Canto II analyzer. Serum cytokine's (TNF- $\alpha$, IL-12p70, IL-6, IFN- $\gamma, \quad$ C-GSF and IL-10) levels in mice were quantitatively measured using the Cytometric Bead Array (CBA) Mouse Inflammation Kit (BD Biosciences, Japan) according to the manufacturer's instructions. Serum chemokines (CXCL-1 and CXCL-2) levels were quantified using ELISA kits (R\&D Systems) according to manufacturer's instructions.

\section{Statistical analysis}

Data were analyzed using GraphPad Software, version 5. Survival curves were constructed by the Kaplan-Meier method and were analyzed using log-rank tests. All other assay data are presented as the mean \pm standard deviation (SD). Statistical significance was determined using the unpaired $t$ test. For all tests, differences were considered to be statistically significant at either $\mathrm{P}<0.01$ or $\mathrm{P}<0.05$.

\section{Results}

Intra-peritoneal administration of LPS at different doses in $\mathrm{BALB} / \mathrm{c}$ mice revealed that $6 \mathrm{mg}$ LPS/ $\mathrm{kg}$ bd. wt. is the LD50 dose 
for this mouse (Figure 1A). In different sets of experiments where LD50 dose of LPS was exposed to wild and DKO mice peritoneum, as a consequence of peritonitis, 15 out of 22 wild and 2 out of 19 DKO (survival percentage in wild $31.8 \%$ vs. $89.5 \%$ in DKO group, $\mathrm{P}=0.001$ ) mice died (Figure 1B).
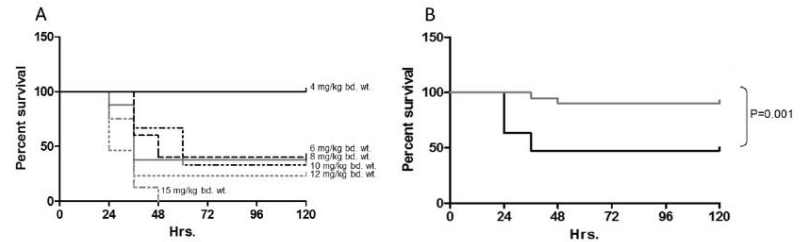

Figure 1: LD50 dose of LPS for wild mice and survival rate in wild and DKO mice after intraperitoneal LPS challenge. (A) Represents survival of wild mice at different dose of LPS. Each group includes minimum 8 mice in two separate sets of experiments. (B) Represents survival percentage of wild (continuous line) and DKO (interrupted line) mice after intraperitoneal administration of LPS at LD50 dose. Each group includes minimum 18 mice in three sets of independent experiments.

Majority of death events occurred within $48 \mathrm{hrs}$. of LPS administration. Particularly, 12 to 30 hrs duration after commencement of peritonitis seems crucial, since before or after this duration mortality rate was low. It indicates that in LPS induced shock event, interactions between inflammatory mediators and host immunity occur within this duration and the interaction of this period ultimately reflects the prognosis of shock. Therefore, we measured various inflammatory parameters at different time points between 0 to $24 \mathrm{hrs}$ of peritonitis. Neutrophils and $\gamma \delta-T$ cells in peritoneal lavage in wild control (non-infected) accounted as $1.14 \%+0.6 \%$ cells $/ \mathrm{ml}$ and $0.36 \%+0.04 \%$ cells $/ \mathrm{ml}$, respectively.
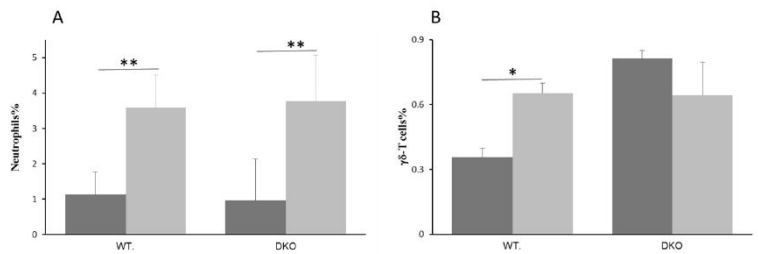

Figure 2: $\gamma \delta-T$ cells and Neutrophils in mice peritoneal lavage. Black and gray bars express mean + SD for control and LPS challenged mice, respectively. (A) Indicates $\gamma \delta$-T cells recruitment status in wild $(n=5)$ and DKO $(n=5)$ mice at $12 \mathrm{hrs}$ of LPS-peritonitis, in compare to control. (B) Indicates neutrophils recruitment status in wild $(n=5)$ and DKO $(n=5)$ mice at $12 \mathrm{hrs}$ of LPS-peritonitis, in compare to control. * denotes $\mathrm{P}<0.05$, ** denotes $\mathrm{P}<0.01$.

Number of these cells accounted as $3.59 \%+1.2 \%$ cells $/ \mathrm{ml}$ $(P<0.05)$ and $0.65 \%+0.03 \%$ cells $/ \mathrm{ml}(P<0.01)$, respectively at 12 hrs of post endotoxic shock (Figure 2). In DKO mice, neutrophils and $\gamma \delta-T$ cells in peritoneal lavage accounted as $0.96 \%+0.9 \%$ cells $/ \mathrm{ml}$ and $0.81 \%+0.05 \%$ cells $/ \mathrm{ml}$, respectively in control and accounted as $3.77 \%+1.3 \%$ cells $/ \mathrm{ml}(\mathrm{P}<0.05)$ and $0.64 \%+0.1 \%$ cells/ml, respectively after $12 \mathrm{hrs}$ of peritonitis (Figure 2). In both wild and DKO mice, after intraperitoneal LPS challenge all cytokines, except IL-10, and chemokines were peaked and declined towards the base line within 24 hrs of infection course. There was a further increase of IL-10 level after $18 \mathrm{hrs}$ of infection in both groups (Figure 3). Serum cytokines and chemokines levels changes by infection in wild and DKO was compared and revealed that changes in IL-6 at 6 , and $12 \mathrm{hrs}$, IFN- $\gamma$ at 12 and 18 hrs, CXCL- 1 at 24 hrs and CXCL- 2 at 3, 6, 12 and $18 \mathrm{hrs}$ of peritonitis were significant (Figure 3 ).
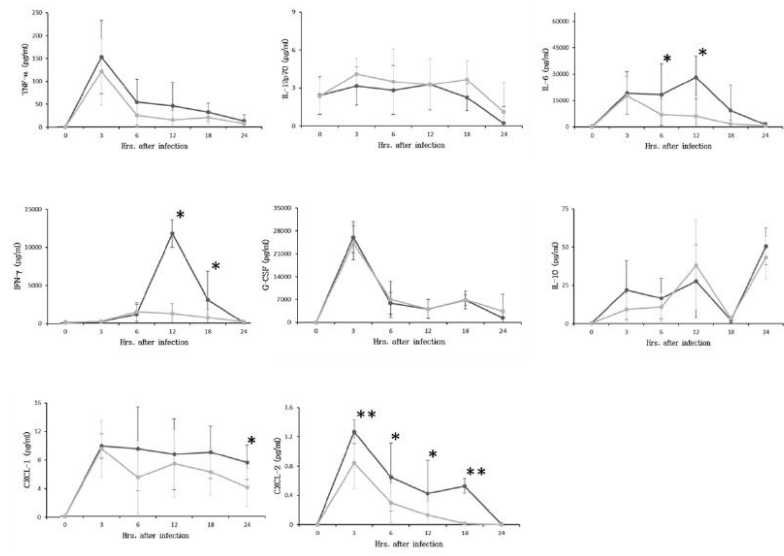

Figure 3: Serum level of inflammatory mediators in wild (black line) and DKO (gray line) mice at different time course of endotoxic shock. Each time point expresses mean+SD serum level of minimum 8 mice by two sets of experiments. ** denotes $\mathrm{P}<0.01$, * denotes $\mathrm{P}<0.05$.

\section{Discussion}

IL-17 response in inflammatory condition was first studied more than 15 years ago [17] and since then its protective function during pathogenic infection and chronic inflammation received priority to study as an emerging therapeutic target in clinical trials. It is generally accepted that IL-17 family are an important modulator of inflammation and protective against invaded pathogens. In current study we prepared LPS induced septic peritonitis in wild and DKO murine and found a higher mortality rate in wild than DKO. The higher mortality was observed in correlation with increased level of serum proinflammatory cytokines and chemokine. There was a significant rise of $\gamma \delta-T$ cell number in peritoneal cavity by LPS in wild models, which is the most potent source for IL-17 release and neutrophil recruitment at the site of infections [18].

A number of cells in both the innate and adaptive immune systems and in non-immune cells [19] produce IL-17A and IL-17F. Both IL-17A and IL-17F have pro-inflammatory properties and act on a broad range of cell types to induce the expression of cytokines, like TGF- $\beta$, TNF- $\alpha$, IL-1 $\beta$, IL-6, IL-21, IL-23, GM-CSF, G- 
CSF, etc. and chemokines, like CXCL1, CXCL2, etc. [20]. In contrast, signals from released cytokines, particularly from IL-1 $\beta$, TGF- $\beta$, IL-6 and IL-23, are required for differentiation of Th17 cells and production of IL-17 cytokines [21]. Over these facts, it is clear that the complex IL-17 regulation in host is difficult to claim entirely known and may differ by various factors. Previous studies showed IL-17AF function in pulmonary bacterial infection is protective [5-10] and this protection may link to increased neutrophil recruitment in infection site, although exact mechanism is unclear [22]. Neutrophil recruitment also reported increased in response to direct exposure of endotoxin in murine airway [23], where survival of the models were not documented. Together these reports suggest that IL-17 mediated neutrophil recruitment in lung is evident with presence of either bacteria or its endotoxin and recruitment is impaired by IL-17 deficiency. Similar to pulmonary infection, in LPS induced acute peritonitis neutrophil recruitment increased in wild mice. Shibata et al. reported same by i.p. E. coli challenge [24]. They also demonstrated impaired neutrophil infiltration by blocking IL-17 activity, but we found DKO mice also significantly recruiting neutrophils in their peritoneum. This discrepancy may be due to the fact that we used genetically depleted mice (II17a $-/-|| 17 f-/-)$ and they used total blocking of IL-17 activity using anti-mIL-17 mAb. However, despite of significant neutrophil infiltration in both wild and DKO models, increased survival rate only in DKO model is interesting and needs further clarification by experiments using real bacteria and bacterial endotoxin in wild and DKO models.

IL-17AF mediated neutrophil recruitment at infection site localizes and engulfs the causative pathogens, is a wellrecognized method for controlling the severity of infection. Neutrophils provide first activated defense line against invaded pathogens. We observed that the number of neutrophil cells in peritoneal cavity increased significantly with LPS administration in both wild and KO models, suggesting neutrophil recruitment in infection site is a common host response. However, LPS administration increased $\gamma \delta-T$ cells only in wild peritoneum. These cells constitute a functionally specialized subset of $T$ lymphocytes which play an important role in linking the innate and adaptive immunity. Growing evidences suggest that these cells provide immunity to infection by recruiting neutrophils at the sites of infection and inflammation. Moreover, the recruitment was reported dominantly done by IL-17 producing $\gamma \delta-T$ cells [2], indicating IL-17 mainly activated by $\gamma \delta-T$ cells in wild mice. In DKO mice, baseline $\gamma \delta-T$ cell level was higher than wild and the level was unchanged by LPS challenge. To our knowledge, this finding is novel in our study that needs to be verified by more investigations. Also the question whether physiological $\gamma \delta-T$ cells function differs from septic stimuli mediated $\gamma \delta-T$ cell function, needs to be evaluated. The evidences of functionally diverse pathogenic and nonpathogenic subpopulation of Th17 cells under specific clinical conditions [11] guide us to assume that $\gamma \delta-T$ cell expression in LPS-peritonitis is responsible for population of pathogenic subsets of Th17 cells in wild mice. Considering all these facts, it is evident in this study that $\gamma \delta-T$ cell mediated neutrophil recruitment by releasing IL-17AF is deleterious, at least in peritonitis.
Acute bacterial peritonitis rapidly commences signs of general septicemia. Besides physical sign-symptoms of systemic sepsis, like lethargy, piloerection, loos bowel, fever, etc., presence of proinflammatory cytokines, chemokines in circulation are the best evidences to determine the status of sepsis condition in a host. In general, increased level of these mediators in blood correlates with the poor outcome of sepsis. Unlike this evidence, we found release of inflammatory cytokines and chemokines in mice circulation in response to LPS induced peritonitis. Among those mediators, several cytokines such as, serum IL-6 and IFN- $\gamma$ and chemokine CXCL-2 (also called MIP-2) level in wild were at significantly higher levels than DKO mice at different time points of shock. It clearly states that comparatively there was low severity of inflammatory reactions in DKO mice than wild, which in consequence showed higher survival rate of DKO mice with peritonitis. On other hand, there was a striking 180 fold rise of serum IFN $-\gamma$ by peritonitis in wild proves that IFN- $\gamma$ production and functions are mainly IL-17AF dependent and this extreme high level may be crucial to determine the shock outcome.

In this study we didn't measure IL-17A, IL-17F or IL-17AF in peritoneum and serum could be considered as limitations. So we can't give a comment whether recruited neutrophils and $\gamma \delta-T$ cells in endotoxin induced peritonitis are capable to produce IL-17 cytokines or not. Rather we described that the control of IL-17AF release is a useful tool for promising outcome in LPS induced peritonitis, at least in mice model. A recent experiment also showed increased survival rate in DKO mice [25], but the investigators used a wide range of LPS dose to introduce peritonitis and we used a single LD50 dose, what is more useful for understanding a specific mechanism in comparative sepsis models. This study provides a comprehensive understanding about the role of IL-17AF functions in specific clinical condition that would be useful for future therapeutic development.

\section{References}

1. Miossec P, Korn T, Kuchroo VK (2009) Interleukin-17 and type 17 helper T cells. N Engl J Med 361: 848-898.

2. Cho JS, Pietras EM, Garcia NC, Ramos RI, Farzam DM, et al. (2010) IL-17 is essential for host defense against cutaneous Staphylococcus aureus infection in mice. J Clin Invest 120: 1762-1773.

3. Ishigame H, Kakuta S, Nagai T, Kadoki M, Nambu A, et al. (2009) Differential roles of interleukin-17A and $-17 \mathrm{~F}$ in host defense against mucoepithelial bacterial infection and allergic responses. Immunity 30: 108-119.

4. Iwakura Y, Nakae S, Saijo S, Ishigame H (2008) The roles of IL-17A in inflammatory immune responses and host defense against pathogens. Immunol Rev 226: 57-79.

5. Kimizuka Y, Kimura S, Saga T, Ishii M, Hasegawa N, et al. (2012) Roles of interleukin-17 in an experimental Legionella pneumophila pneumonia model. Infect Immun 80:1121-1127.

6. Zhou X, Chen Q, Moore J, Kolls JK, Halperin S, et al. (2009) Critical role of the interleukin-17/interleukin-17 receptor axis in regulating host susceptibility to respiratory infection with Chlamydia species. Infect Immun 77: 5059-5070.

7. Wu Q, Martin RJ, Rino JG, Breed R, Torres RM, et al. (2007) IL-23dependent IL-17 production is essential in neutrophil recruitment 
and activity in mouse lung defense against respiratory Mycoplasma pneumoniae infection. Microbes Infect 9: 78-86.

8. Zhang X, Gao L, Lei L, Zhong Y, Dube P, et al. (2009) A MyD88dependent early IL-17 production protects mice against airway infection with the obligate intracellular pathogen Chlamydia muridarum. J Immunol 183: 1291-1300.

9. Ye P, Garvey PB, Zhang P, Nelson S, Bagby G, et al. (2001) Interleukin-17 and lung host defense against Klebsiella pneumoniae infection. Am J Respir Cell Mol Biol 25: 335-340.

10. Kudva A, Scheller EV, Robinson KM, Crowe CR, Choi SM, et al. (2011) Influenza A inhibits Th17-mediated host defense against bacterial pneumonia in mice. J Immunol 186:1666-1674.

11. Jin W, Dong C (2013) IL-17 cytokines in immunity and inflammation. Emerg Microbes Infect 2: e60.

12. Barretti P, Doles JV, Pinotti DG, El Dib R (2014) Efficacy of antibiotic therapy for peritoneal dialysis-associated peritonitis: a proportional meta-analysis. BMC Infect Dis 14: 445.

13. Ogiku $M$, Kono $H$, Hara $M$, Tsuchiya $M$, Fujii H (2012) Interleukin-17A plays a pivotal role in polymicrobial sepsis according to studies using IL-17A knockout mice. J Surg Res 174: 142-149.

14. Li J, Zhang Y, Lou J, Zhu J, He M, et al. (2012) Neutralization of peritoneal IL-17A markedly improves the prognosis of severe septic mice by decreasing neutrophil infiltration and proinflammatory cytokines. PLoS One 7: e46506.

15. Horai R, Nakajima A, Habiro K, Kotani M, Nakae S, et al. (2004) TNF- $\alpha$ is crucial for the development of autoimmune arthritis in IL-1 receptor antagonist-deficient mice. J Clin Invest 114: 1603-1611.

16. Nakae S, Komiyama $\mathrm{Y}$, Nambu A, Sudo K, Iwase M, et al. (2002) Antigen-specific $T$ cell sensitization is impaired in IL-17-deficient mice, causing suppression of allergic cellular and humoral responses. Immunity 17: 375-387.
17. Miossec $\mathbf{P}$ (2007) Interleukin-17 in fashion, at last: ten years after its description, its cellular source has been identified. Arthritis Rheum 56: 2111-2115.

18. Sabbione F, Gabelloni ML, Ernst G, Gori MS, Salamone G, et al. (2014) Neutrophils suppress $\gamma \delta$ T-cell function. Eur J Immunol 44: 819-830.

19. Yamada H (2010) Current perspectives on the role of IL-17 in autoimmune disease. J Inflam Res 3: 33-44.

20. Maddur MS, Miossec P, Kaveri SV, Bayry J (2012) Th17 cells: biology, pathogenesis of autoimmune and inflammatory diseases, and therapeutic strategies. Am J Pathol 181: 8-18.

21. Caccamo N, Mendola CL, Orlando V, Meraviglia S, Todaro M, et al. (2011) Differentiation, phenotype and function of interleukin-17producing human Vg9Vd2 T cells. Blood 118: 129-138.

22. Tsai HC, Velichko S, Hung LY, Wu R (2013) IL-17A and Th17 cells in lung inflammation: an update on the role of Th17 cell differentiation and IL-17R signaling in host defense against infection. Clin Dev Immunol 2013: 267971.

23. Miyamoto $M$, Prause $O$, Sjöstrand $M$, Laan $M$, Lötvall J, Lindén $A$ (2003) Endogenous IL-17 as a mediator of neutrophil recruitment caused by endotoxin exposure in mouse airways. J Immunol 170: 4665-4672.

24. Shibata K, Yamada H, Hara H, Kishihara K, Yoshikai Y (2008) Resident $V \delta 1+\gamma \delta$ T Cells Control Early Infiltration of Neutrophils after Escherichia coli Infection via IL-17 Production. J Immunol 181: 2071-2075.

25. Shimura E, Shibui A, Narushima S, Nambu A, Yamaguchi $S$, et al. (2014) Potential role of myeloid cell/eosinophil-derived IL-17 in LPS-induced endotoxin shock. Biochem Biophys Res Commun 453: 1-6. 